International Journal of Artificial Intelligence \& Applications (IJAIA), Vol. 4, No. 4, July 2013

\title{
Data Mining Un-Compressed Images from cloud with Clustering Compression technique using Lempel-Ziv-Welch
}

\author{
${ }^{1}$ C. Parthasarathy ${ }^{2}$ K.Srinivasan and ${ }^{3}$ R.Saravanan \\ Assistant Professor, ${ }^{1,2,3}$ Dept. of I.T, SCSVMV University, Enathur, Kanchipuram, \\ Pin-631 561 \\ ${ }^{1}$ sarathy286089@rediffmail.com ${ }^{2}$ kadamsrini21@gmail.com \\ ${ }^{3}$ saravanan_kpm1983@yahoo.co.in
}

\begin{abstract}
Cloud computing is a highly discussed topic in the technical and economic world, and many of the big players of the software industry have entered the development of cloud services. Several companies' and organizations wants to explore the possibilities and benefits of incorporating such cloud computing services in their business, as well as the possibilities to offer own cloud services. We are going to mine the un-compressed image from the cloud and use k-means clustering grouping the uncompressed image and compress it with Lempel-ziv-welch coding technique so that the un-compressed images becomes error-free compression and spatial redundancies.
\end{abstract}

\section{KEYWORDS}

Cloud, Compression, Image processing, Clustering

\section{INTRODUCTION}

In cloud computing, the word cloud is used as a metaphor for "the Internet" so the phrase cloud computing means "a type of Internet-based computing," where different services -- such as servers, storage and applications -- are delivered to an organization's computers and devices through the Internet.

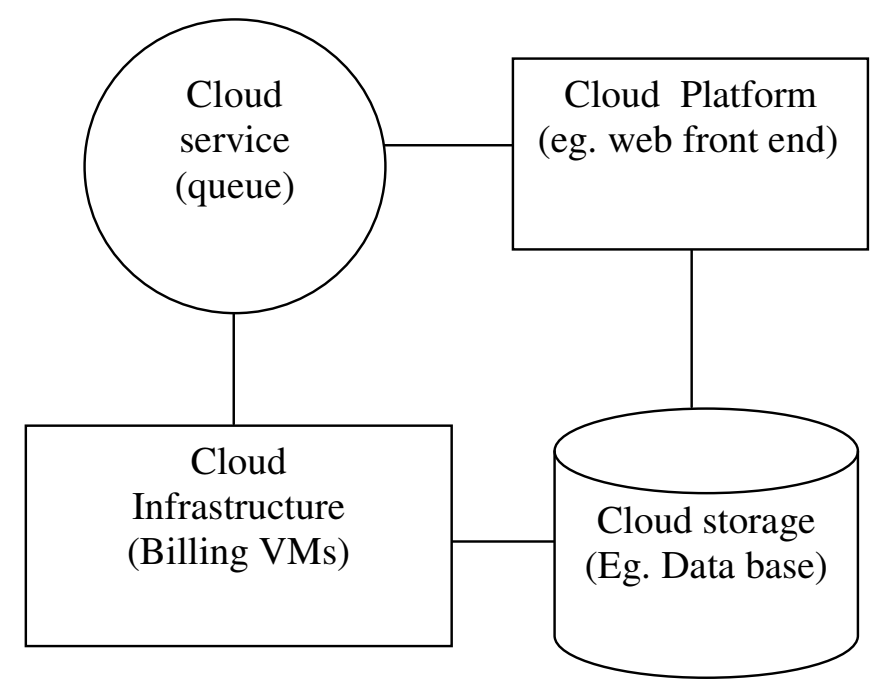

DOI : 10.5121/ijaia.2013.4413

Figure 1. Cloud Computing Model 
Clustering can be considered the most important unsupervised learning technique; so, as every other problem of this kind, it deals with finding a structure in a collection of unlabeled data. Clustering is "the process of organizing objects into groups whose members are similar in some way". A cluster is therefore a collection of objects which are "similar" between them and are "dissimilar" to the objects belonging to other clusters. The typical requirements of clustering in data mining are 1 . Scalability 2 . Ability to deal with different types of attributes 3 . Discovery of clusters with arbitrary shape 4 . Minimal requirements for domain knowledge to determine input parameters 5. Ability to deal with noisy data 6 . Incremental clustering and insensitivity to the order of input records. 7. High dimensionality. Clustering is also called data segmentation because clustering partitions large data sets into groups according to their similarity.

\section{K-MEANS METHOD}

In this cloud we are going to cluster the un-compressed Image by centroid based technique: The $\mathrm{K}$-means algorithm takes input parameter and partitions a set of $\mathrm{n}$-objects into k clusters so that the resulting intra-cluster similarity is high but the inter-cluster similarity is low. K-Means algorithm proceeds as it randomly selects $\mathrm{k}$ of the objects, an object is assigned to the cluster it is the most similar, based on the distance between the object and the cluster mean. It Computes the new mean for each cluster it iterates until the criterion function converges.

The square-error criterion

$$
\begin{aligned}
& \mathrm{k} \\
& \mathrm{E}=\sum \sum \text { | } \mathrm{p} \text {-mil } 2 \text { where } \mathrm{E} \text { is the sum of the square error for all } \\
& \text { objects in } \quad \mathrm{i}=1 \quad \mathrm{peC} i
\end{aligned}
$$

the data set; $\mathrm{p}$ is the point in space representing a given object and mi is the mean of cluster $\mathrm{Ci}$

Algorithm: The k-means algorithm for partitioning, where each cluster's center is represented by the mean value of the objects in the cluster.

Input :

\section{Method:}

$\mathrm{k}$ : the number of clusters

D: a data set containing $n$ objects

(1) arbitrarily choose $\mathrm{k}$ objects from $\mathrm{D}$ as the initial cluster centers Repeat Assign each object to the cluster to which the object is the most similar Based on the mean value of the objects in the cluster; Update the cluster means, i.e., calculate the mean value of the object for Each cluster; Until no change.

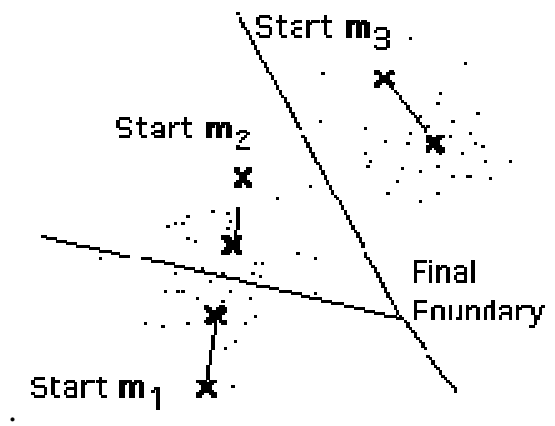

Figure 2. shows the mean $\mathrm{m} 1, \mathrm{~m} 2$ is far away from the final boundary 
International Journal of Artificial Intelligence \& Applications (IJAIA), Vol. 4, No. 4, July 2013

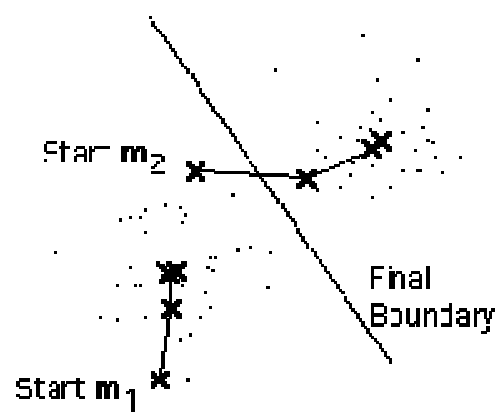

Figure 3. shows how the means $\mathbf{m} 1$ and $\mathbf{m} 2$ move closer to the final boundary

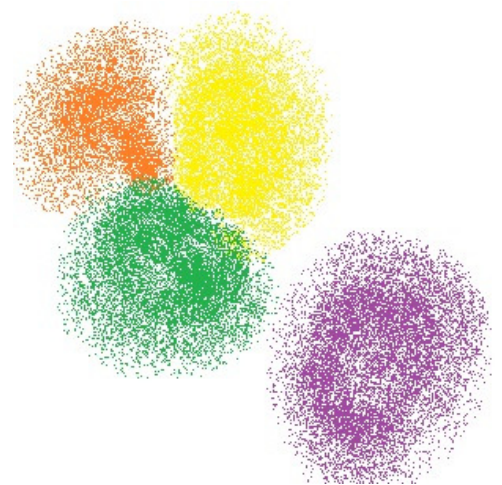

Figure 4. shows different types of clusters

After the algorithm finishes, it produces these outputs:

- A label for each data point

- The center for each label

A label can be considered as "assigning a group". For example, in the above image you can see four "labels". Each label is displayed with a different colour. All yellow points could have the label 0 , orange could have label 1 , etc.
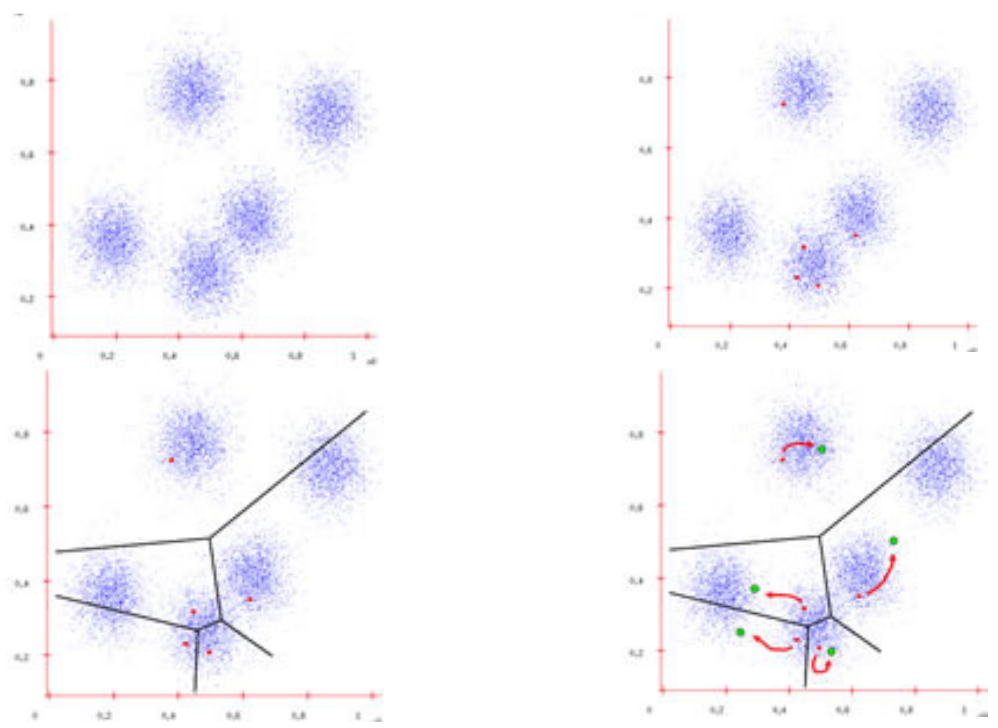


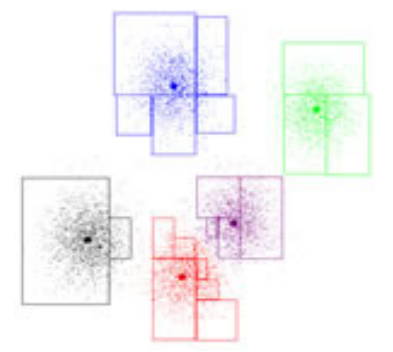

Figure 5. Grouping different set of uncompressed images

Step 0: Get the dataset

As an example, We will be using the data points on the left. We'll assume $\mathrm{K}=5$. And it's apparent that this dataset has 5 clusters: three spaced out and two almost merging.

Step 1: Assign random centers

The first step is to randomly assign $\mathrm{K}$ centers. We have marked them as red points in the image. Note how they are all concentrated in the two "almost merging" clusters. These centers are just an initial guess. The algorithm will iteratively correct itself. Finally, these centers will coincide with the actual center of each cluster.

Step 2: "Own" datapoints

Each datapoint checks which center it is closest to. So, it "belongs" to that particular center. Thus, all centers "own" some number of points.

Step 3: Shift the centers

Each center uses the points it "owns" to calculate a new center. Then, it shifts itself to that center.

If the centers actually shifted, we again go to Step 3. If not, then the centers are the final result.

We proceed to the next step

Now that the centers do not move, you can use the centers.

\section{SCOPE OF THE RESEARCH}

We know about the term compression ratio. This means as:

Compression Ratio, $\mathrm{Cr}=(($ Data size of original message $) /($ Data size of Encoded message $))$

Now-a-days, compression ratio is a great factor in transmission of data. By this research we can have a better solution about how to make compression ratio higher, because data transmission mostly depends on compression ratio. Images transmitted over the world wide web here data compression is important. Suppose we need to download a digitized color photograph over a computer's $33.6 \mathrm{kbps}$ modem. If the image is not compressed (a TIFF file, for example), it will contain about 600 kbytes of data. If it has been compressed using a LSW CODING technique (such as used in the GIF format), it will be about one-half this size, or 300 kbytes. If LSW CODING TECHNIQUE has been used (a JPEG file), it will be about 50 kbytes. The point is, the download times for these three equivalent files are 142 seconds, 71 seconds, and 12 seconds, respectively. Digitized photographs, while GIF is used with drawn images, such as company logos that have large areas of a single color.

Disadvantage of Lossless Compression

Lossless compression, refers to the process of encoding data more efficiently so that it occupies fewer bits or bytes but in such a way that the original data can be reconstructed, bit-for-bit, when 
International Journal of Artificial Intelligence \& Applications (IJAIA), Vol. 4, No. 4, July 2013

the data is decompressed. The advantage of lossless encoding techniques is that they produce an exact duplicate of the original data but they also have some disadvantages when compared to Lossy encoding techniques.

\section{COMPRESSION RATIO}

Lossless encoding techniques cannot achieve high levels of compression. Few lossless encoding techniques can achieve a compression ratio higher than 8:1 which compares unfavorably with socalled lossy encoding techniques. Lossy encoding techniques -- which achieve compression by discarding some of the original data -- can achieve compression ratios of 10:1 for audio and 300:1 for video with little or no perceptible loss of quality. According to the New Biggin Photography Group a 1,943 by 1,702 pixel 24-bit RGB color image with an original size of 9.9 megabytes can only be reduced to 6.5 megabytes using the lossless PNG format but can be reduced to just 1 megabyte using the lossy JPEG format.

\section{TRANSFER TIME}

Any application that involves storing or distributing digital images, or both, presupposes that these operations can be completed in a reasonable length of time. The time needed to transfer a digital image depends on the size of the compressed image and as the compression ratios that can be achieved by lossless encoding techniques are far lower than lossy encoding techniques, lossless encoding techniques are unsuitable for these applications.

\subsection{Lempel-Ziv-Welch (Lzw) Coding Technique}

LZW compression is named after its developers, A. Lempel and J. Ziv, with later modifications by Terry A. Welch. It is the foremost technique for general purpose data compression due to its simplicity and versatility. Typically, you can expect LZW to compress text, executable code, and similar data files to about one-half their original size. LZW also performs well when presented with extremely redundant data files, such as tabulated numbers, computer source code, and acquired signals. Compression ratios of 5:1 are common for these cases.

LZW compression uses a code table, as illustrated in Fig. 6. A common choice is to provide 4096 entries in the table. In this case, the LZW encoded data consists entirely of 12 bit codes, each referring to one of the entries in the code table. Un compression is achieved by taking each code from the compressed file, and translating it through the code table to find what character or characters it represents. Codes 0-255 in the code table are always assigned to represent single bytes from the input file. For example, if only these first 256 codes were used, each byte in the original file would be converted into 12 bits in the LZW encoded file, resulting in a $50 \%$ larger file size. During un compression, each 12 bit code would be translated via the code table back into the single bytes. Of course, this wouldn't be a useful situation.

\section{ADVANTAGE OF LZW COMPRESSION}

The LZW compression can compress executable code, text, and similar data files to almost onehalf of their original size. It usually uses single codes to replace strings of characters, thereby compressing the data. LZW also gives a good performance when extremely redundant data files are presented to it like computer source code, tabulated numbers and acquired signals. The common compression ratio for these cases is almost in the range of 5:1. 


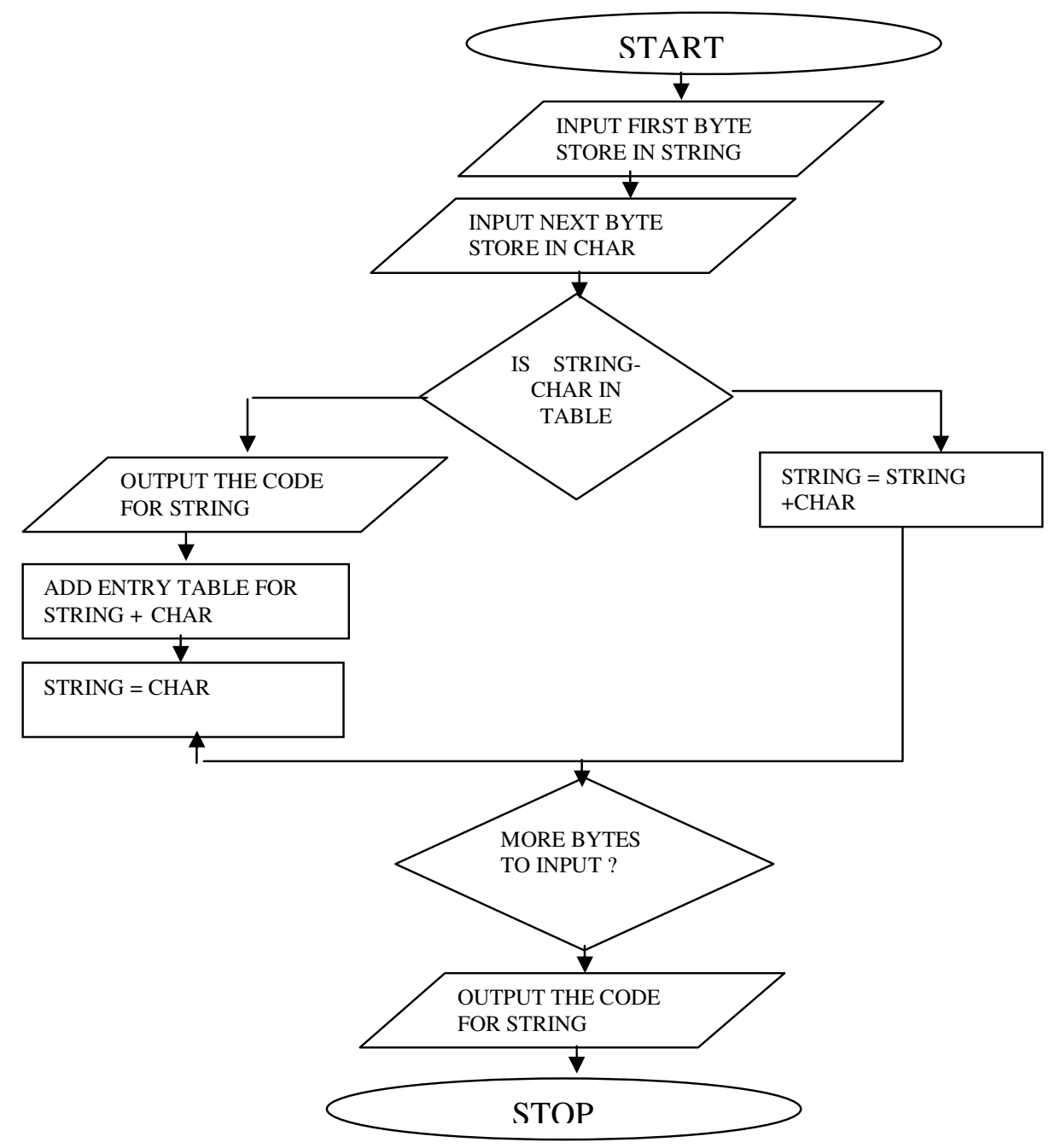

Figure 6 - Flow chart for LZW coding compression

Many methods of LZW coding compression have been developed; however, a family of techniques called transform compression has proven the most valuable. The best example of transform compression is embodied in the popular JPEG standard of image encoding. JPEG is named after its origin, the Joint Photographers Experts Group. LZW compression is different from other common compression algorithms (e.g. Huffman encoding) in that it does not require an initial processing of the data file to determine the codes to be used. Instead, the code table is generated "on the fly" at the same time that the file is compressed. The algorithm operates so that at each basic step, either the code for a sequence of values already exists in the table, or the code for a new sequence is generated and inserted into the table. Another interesting characteristic of LZW compression is that it is not necessary to send a full code table along with the compressed file. Only a table of the individual colors that exist in the image is needed. Then codes for sequences of colors are generated during the decompression process such that the codes are always available in the table when they are needed. 
International Journal of Artificial Intelligence \& Applications (IJAIA), Vol. 4, No. 4, July 2013

The first step in LZW is initialization of the code table to include all the colors in the image file. For an 8-bit image, this would be up to 256 colors. If all colors were used, the codes would be the values from 0 through 255 , each code requiring eight bits. The algorithm proceeds by processing the pixels in the image file from left to right and top to bottom of the image. The basic idea of the algorithm is that strings of colors are put into the code table as they are encountered. Grouping strings of colors together into a single code results in compression.

The basic algorithm for LZW compression is given below. In the pseudocode that follows, pixelString is a sequence of pixel values. pixel = next pixel value means "read the next pixel out of the image file." pixelString + pixel means "take the current pixelString value and concatenate pixel onto the end of it."

algorithm LZW

/*Input: A bitmap image

Output: A table of the individual colors in the image and a compressed version of the file Note that + is concatenation*/ \{

initialize table to contain the individual colors in bitmap

pixelString $=$ first pixel value

while there are still pixels to process \{

pixel $=$ next pixel value

stringSoFar $=$ pixelString + pixel

if stringSoFar is in the table then

pixelString $=$ stringSoFar

else \{

output the code for pixelString

add stringSoFar to the table

pixelString $=$ pixel

\}

\}

output the code for pixelString

\}

Consider an simplified problem a string BABAABAAA use the LZW Algorithm to compress the string.

TABLE-1 EXAMPLE WORK FOR COMPRESSION

\begin{tabular}{|c|c|c|c|}
\hline ENCODER OUTPUT & $\begin{array}{c}\text { OUTPUT } \\
\text { REPRESENTING }\end{array}$ & $\begin{array}{c}\text { STRING CODE } \\
\text { WORD }\end{array}$ & TABLE STRING \\
\hline 66 & $\mathrm{~B}$ & 256 & $\mathrm{BA}$ \\
\hline 65 & $\mathrm{~A}$ & 257 & $\mathrm{AB}$ \\
\hline 256 & $\mathrm{BA}$ & 258 & $\mathrm{BAA}$ \\
\hline 257 & $\mathrm{AB}$ & 259 & $\mathrm{ABA}$ \\
\hline 65 & $\mathrm{~A}$ & 260 & $\mathrm{AA}$ \\
\hline
\end{tabular}


International Journal of Artificial Intelligence \& Applications (IJAIA), Vol. 4, No. 4, July 2013

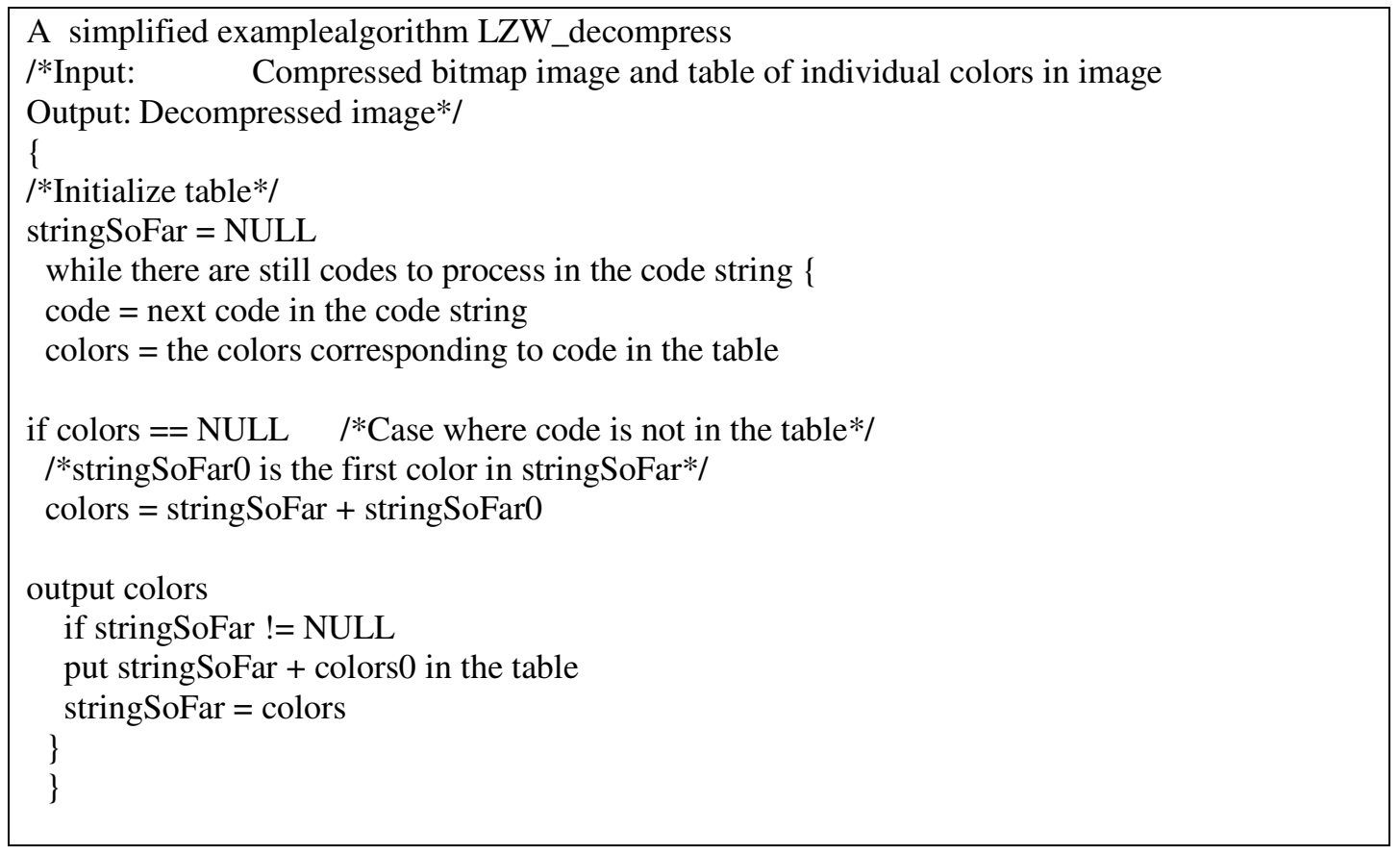

Basic LZW Decompression Algorithm

A simplified example for the string BABAABAAA the compressed code words are $<66><65><256><257><258><259><260>$ using the code word we are going to de-compress the information

TABLE -2 EXAMPLE WORK FOR DE-COMPRESSION

\begin{tabular}{|c|c|c|}
\hline Encoder output string & String table code-word & String \\
\hline B & & \\
\hline A & 256 & BA \\
\hline BA & 257 & AB \\
\hline AB & 258 & BAA \\
\hline A & 259 & ABA \\
\hline AA & 260 & AA \\
\hline
\end{tabular}




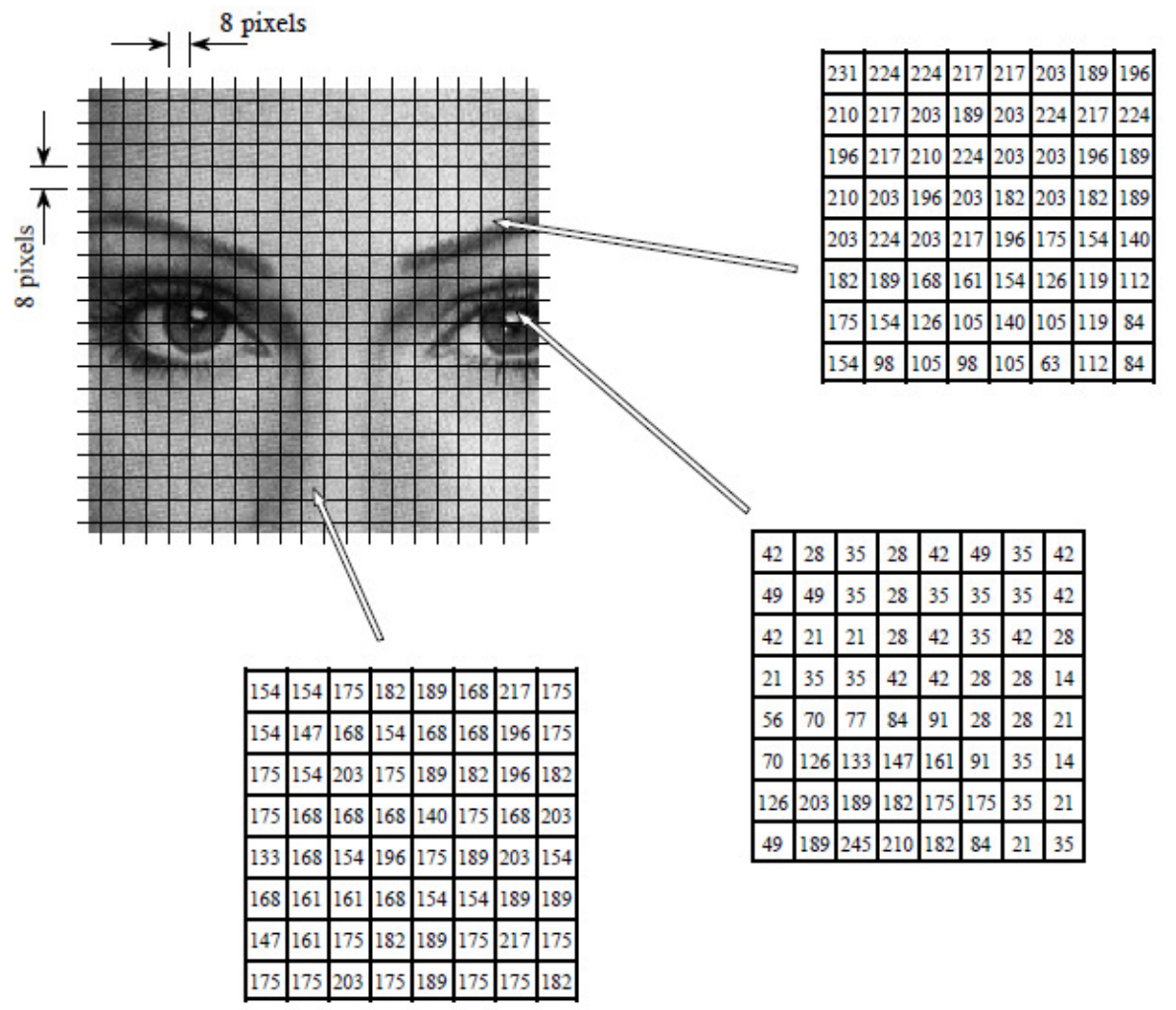

Figure 7. JPEG IMAGE DIVISION

JPEG image division. JPEG transform compression starts by breaking the image into $8 \times 8$ groups, each containing 64 pixels. Three of these $8 \times 8$ groups are enlarged in this figure, showing the valuesof the individual pixels, a single byte value between 0 and 255 .

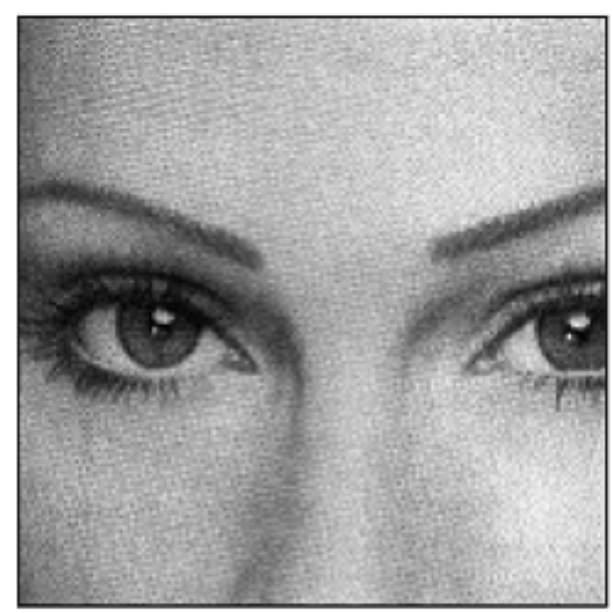

A. Original image 
International Journal of Artificial Intelligence \& Applications (IJAIA), Vol. 4, No. 4, July 2013

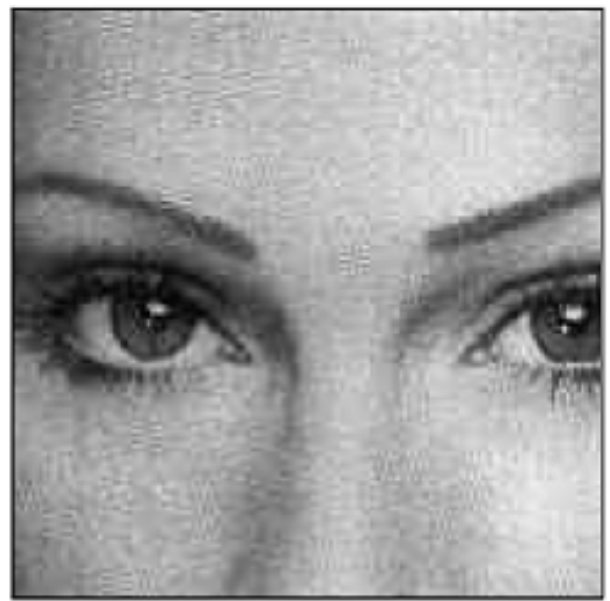

B. With 10:1 Compression

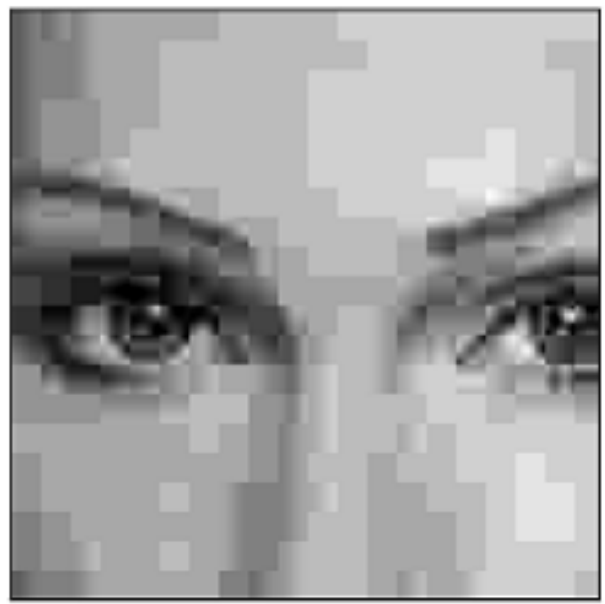

C. With 45:1 Compression

Figure 8 Example of JPEG distortion. Figure(a) shows the original image, while (b) and (c) shows restored images using compression ratios of 10:1 and 45:1, respectively. The high compression ratio used in (c) results in each $8 \mathrm{X} 8$ pixel group being represented by less than 12 bits.

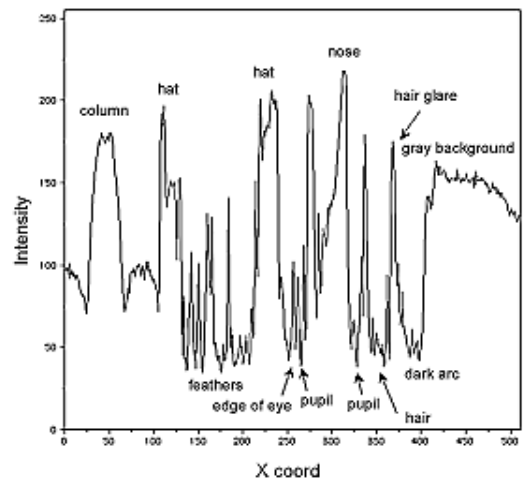

Figure 9. A plot of the intensity data of line 266 of the original (uncompressed) image 


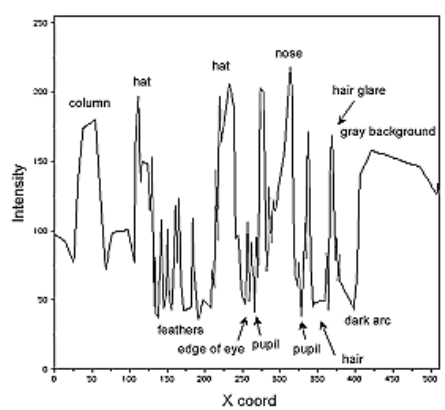

Figure 10. A plot of the intensity data of line 266 of the $1.0 \mathrm{bpp}$ NSI compressed image.

\section{CONCLUSION}

Image compression is a topic of much importance and employed in many applications. Methods of Image compression have been studied for almost four decades. An enhanced scaling algorithm was devised. This algorithm utilized the proximity of the sample points chosen to each other to detect edges This paper has provided an overview of Image compression methods of general utility. The algorithms have been evaluated in terms of the amount of compression they provide, algorithm efficiency, and susceptibility to error. While algorithm efficiency and susceptibility to error are relatively independent of the characteristics of the source ensemble, the amount of compression achieved depends upon the characteristics of the source to a great extent.

\section{REFERENCES}

[1] “Digital Image Processing” Second paper Edition-(2006) ,Rafael C. Gonzalez and Richard E. Woods. Pages: 411, 440-442, 459.

[2] "Lecture on Data Compression", Patrick Karlsson Patrick. karlsson@ @b.uu.se

[3] "Error-Resilient LZW Data Compression" , YonghuiWu Stefano Lonardi WojciechSzpankowski, Proceedings of the Data Compression Conference IEEE(DCC'06) 0-7695-2545-8 /06 (2006)

[4] "LZW Data Compression" Mark Nelson's from the October, 1989 issue of Dr. Dobb's Journal.

[5] "A fast binary template matching algorithm for document image data compression in Pattern Recognition”, Holt, M.J. (1988) J. Kittler (ed.) (Proc. Int. Conf.,Cambridge). Springer Verlag, Berlin.

[6] "Compression of black-white images with arithmetic coding," Langdon, G.G. and Rissanen, J. (1981)June IEEE Trans Communications COM-29(6): 858-867.

[7] “Two level context based compression of binary images”, in Proc. DCC'91, J.A. Storer and J.H. Reif (eds.), Moffat, A. IEEE Computer Society Press, Los Alamitos (1991) pp. 382-391.

[8] "Combined symbol matching facsimile data compression system", Pratt, W.K., Capitant, P.J., Chen, W.H.,Hamilton, E.R. and Wallis, R.H. (1980) July Proc IEEE 68(7): 786-796;.

[9] “Textual Image Compression” in Proc.DCC'92, J.A. Storer and M. Cohn (eds.),Witten, I.H., Bell, Harrison, James and Moffat, A. IEEE Computer Society Press, Los Alamitos, CA. (1992) pp. 42-51.

[10] "A universal algorithm for sequential data compression", ZIV, J. AND LEMPEL, A.(197I)IEEE Trans. Inform. Theory 23, 3, 337-343.

[11] “Text Compression English word” T. C. Bell, J. G. Cleary, and I. H. Witten,Cliffs:N. J. PrenticeHall, 1990.

[12] “The LEMPEL-ZIV-WEL (LZW) Data Compression Algorithm for Packet Radio", Kinsner and R H. Greenfield -IEEE

[13] "Enhanced LZW (Lempel-Ziv-Welch) Algorithm with Binary Search to Reduce Time Complexity for Dictionary Creation in Encoding and Decoding", published Proceedings of international conference(ICICIC 2012) NISHAD PM and Dr. N. NALAYINI -PSG tech 5- January 2012.

[14] "PPM performance with BWT Complexity: A fast and effective data compression algorithm", M. Effros,Proceedings of the IEEE, 88(11), 1703-1712, (2000).

[15] "Compression of two-dimensional data", A. Lempel and J. Ziv, IEEE Transactions on Information Theory, 32(1), 2-8, (1986). 